\title{
Haptic sensitivity in needle insertion: the effects of training and visual aid
}

\author{
Emilio Loren Roth Monzon $^{*} \quad$ Amine Chellali $^{\dagger} \quad$ Cedric Dumas ${ }^{\top} \quad$ Caroline G.L. Cao ${ }^{\star}$ \\ (*)Institut de Recherche en Communications et Cybernétique de Nantes, France \\ (†) Ecole des Mines de Nantes, France \\ ( ) Commonwealth Scientific and Industrial Research Organisation, Australia \\ (t)Tufts University, USA \\ E-mail:emilic@gmail.com,Amine.Chellali@mines-nantes.fr, \\ Cedric.Dumas@csiro.au,Caroline.Cao@tufts.edu
}

\begin{abstract}
This paper describes an experiment conducted to measure haptic sensitivity and the effects of haptic training with and without visual aid. The protocol for haptic training consisted of a needle insertion task using dual-layer silicon samples. A visual aid was provided as a multimodal cue for the haptic perception task. Results showed that for a group of novices (subjects with no previous experience in needle insertion), training with a visual aid resulted in a longer time to task completion, and a greater applied force, during post-training tests. This suggests that haptic perception is easily overshadowed, and may be completely replaced, by visual feedback. Therefore, haptic skills must be trained differently from visuomotor skills.
\end{abstract}

\section{Introduction}

In several medical procedures clinicians depend on their haptic perception abilities to insert needles in the patient; for example, in the administration of drugs and in radiological percutaneous needle insertions to perform biopsies as shown in figure 1:

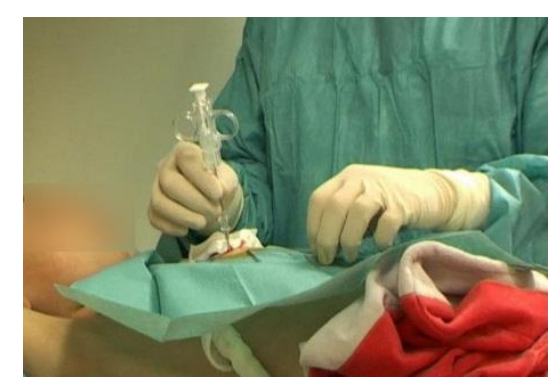

Fig. 1: Needle insertion in a biopsy procedure
To reach the target tissue, clinicians must pierce through several layers of different types of tissue; the precision of this insertion depends on the clinician's knowledge of anatomy, spatial representation and haptic perception. This knowledge and perception ability is gained through years of experience in clinical practice.

Several studies Error! Reference source not found.[2] have shown that surgeons are better at haptic perception tasks (measured through applied forces and time needed for the task completion) than subjects without any previous training. In all cases, visual cues can improve the haptic perception task performances by diminishing the error rate.

The visual cue that is given to the subjects can be of different types. Zhou, Perrault, Schwaitzberg and Cao [2][3] used the image coming from an endoscope to give information about the position of the tool and the amount of force applied by subjects; whereas Gerovich [1] used a simulation in which the user could see the different layers of tissue being tested and the real-time position of the needle in a needle-insertion task.

\section{Objectives and hypotheses}

The goal of this research is to examine the haptic sensitivity of experts and novices in a needle insertion task. The objectives of the study are to quantify the effects of training. In addition, the benefit of a visual aid used during training is investigated. It is hypothesized that training will improve the participants' performance. A visual aid is expected to improve performance, both during the training sessions and after training when the visual aid is not in use.

This is an Open Access article distributed under the terms of the Creative Commons Attribution-Noncommercial License 3.0, which permits unrestricted use, distribution, and reproduction in any noncommercial medium, provided the original work is properly cited. 


\section{Methodology}

The previous hypotheses were tested in a controlled experiment. A visual aid was designed to provide real-time information about the actual forces applied by the participants.

\subsection{Simulated task}

The task was designed to simulate needle handling during anesthetic needle insertion. Tissue was simulated using silicon samples (Figure 2). The participants were instructed to perforate the silicone using an anesthetic needle (Figure 3) until they reached the middle layer of the dual-layered gel samples. Depending on the experimental conditions, the task was performed with or without a visual aid which consisted of a real-time display for the forces applied by the participants on the needle.

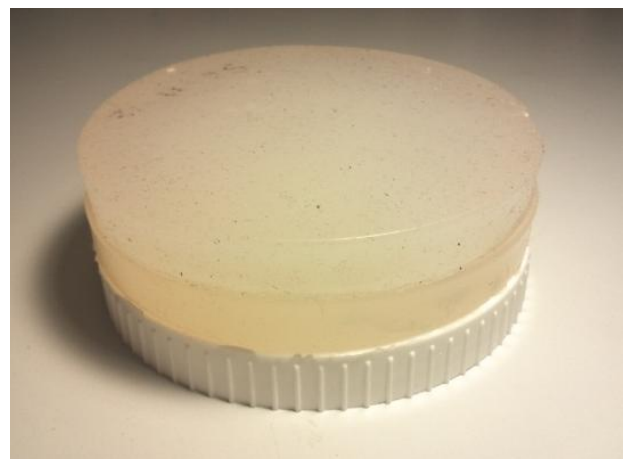

Fig. 2: The dual layer silicon sample

The gel samples were made using RTV silicone (Room Temperature Vulcanizing) EC00 [4]. The compliance of the silicon was controlled by changing the dilution required for the creation of the samples and measured by doing a mechanical compression test with an Instron compression tester.

The top silicon layer was softer than the bottom layer. The difference in compliance between the two layers was always greater than the Just Noticeable Difference in compliance (JND, is the "sensitivity" of the human haptic system to discriminate between different compliances [5]) reported for similar silicon samples [6]. The difference between the top and the bottom layers was regulated to create two values of constant difference in compliance to allow a different level of haptic perception during the trials.

The participants were instructed to halt penetration when they reached the middle layer just before penetrating the second layer, simulating a needle insertion task in anesthetic needle insertion when the desired point is reached.

\subsection{Apparatus used}

To comply with the anesthetic needle insertion, a $22^{\circ}$ bevel-tip needle was used. An ATI Nano 17 force sensor which has 6 degrees of freedom (3 force and 3 torque) was mounted to the handle of the needle to measure the instantaneous force that was felt by the user during the needle insertion. The force sensor had an ergonomic grasping device for ease of access of the haptic needle.

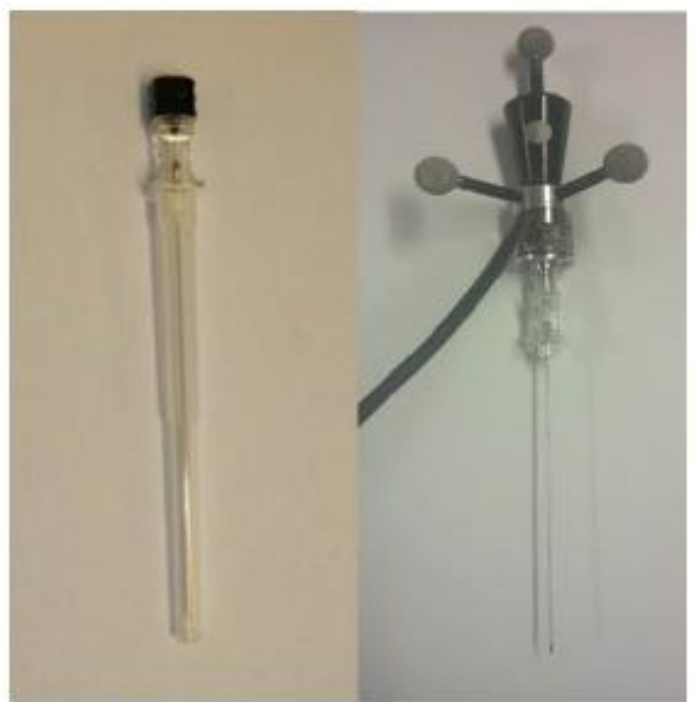

Fig. 3: Anesthetic (left) and instrumented (right) needles for position and force/torque measurement

The position of the needle was tracked using 5 OptiTrack infrared cameras (NaturalPoint Inc.) positioned to cover the volume of movement used by the participant during his examination of the samples and by adding a total of 4 markers in the ergonomic part of the haptic needle.

To prevent participants from learning the physical position of the tissue samples and their compliance difference, a Lazy Susan was made with eight different heights which allowed a fast sample change and height variation during the experiment. The devise was placed in a box (Figure 4) that had a $0.6 \mathrm{~cm}$ hole cut into the top surface. This hole served as a guide for the needle insertion. This prevented the participants from seeing the position of the target sample, forcing them to rely only on haptic perception during the trials without a visual aid.

For the trials with a visual aid, a computer screen was placed in front of the participants so that they could match their haptic perception with the force profile displayed on the monitor (see Figure 4). Data collection was managed by a real-time program in $\mathrm{C}++$ using Nokia Qt GUI system. 


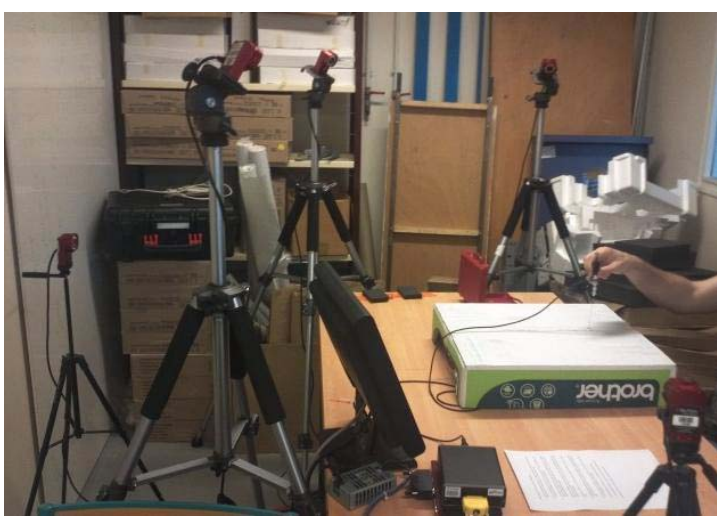

Fig. 4: Experimental setup

\subsection{Experimental Design}

Sixteen subjects participated in this experiment and were divided into 4 groups. Participants marked as experts were clinicians that reported experience with needles. The novice subjects had no previous experience with needles. The participants' setup and division can be seen in Table 1 .

All the participants performed a pre test session.

A total of 8 dual layer silicon samples were used per trial, which were positioned at different heights and would be easily interchangeable to minimize the subject time of the experiment. Each participant performed a total of 6 trials (3 with a visual aid, and 3 without a visual aid). The presentation of the samples to the user was randomized, while the visual aid condition was counterbalanced.

After the pre test, two novice groups performed a training session, one group with visual aid and the other group without visual aid. The visual aid consisted of a real-time plot of the force applied by the needle versus the time, emphasizing the notion of puncturing and crossing the gel layers by the needle and when the middle layer was reached.

\begin{tabular}{|c|c|c|c|}
\hline Participants & $\begin{array}{c}1^{\text {st }} \text { Session: } \\
\text { Pre Test }\end{array}$ & $\begin{array}{l}\text { Training } \\
\text { Session }\end{array}$ & $\begin{array}{c}3^{\text {rd }} \\
\text { Session: } \\
\text { Post Test }\end{array}$ \\
\hline 4 Experts & $\begin{array}{c}48 \\
\text { (8 samples } \\
\text { x } 6 \text { trials) }\end{array}$ & No & $\begin{array}{c}48 \\
(8 \times 6)\end{array}$ \\
\hline 4 Novices & $\begin{array}{c}48 \\
(8 \times 6)\end{array}$ & $\begin{array}{c}\text { Visual cue } \\
\text { 1h/8 samples } \mathrm{x} \\
10 \text { trials }\end{array}$ & $\begin{array}{c}48 \\
(8 \times 6)\end{array}$ \\
\hline 4 Novices & $\begin{array}{c}48 \\
(8 \times 6)\end{array}$ & $\begin{array}{l}\text { No visual cue } \\
1 \mathrm{~h} / 8 \text { samples } \mathrm{x} \\
10 \text { trials }\end{array}$ & $\begin{array}{c}48 \\
(8 \times 6)\end{array}$ \\
\hline 4 Novices & $\begin{array}{c}48 \\
(8 \times 6)\end{array}$ & No & $\begin{array}{c}48 \\
(8 \times 6)\end{array}$ \\
\hline
\end{tabular}

After the training period, a test was carried out again, counterbalancing the use of the visual aid. This post-test was also carried out for those subjects who did not receive training, to see if with they would also present variations in their haptic perception after a week of their normal activities.

The totality of the experiment was carried out in a 7-day period for each participant. The pre-test was done on Day One in a 30-min session. Training would occur 3 days later. The post test was done on Day Seven, in a 20-30 min session.

\section{Results}

Preliminary results show that the training with visual cues does not significantly improve the time to task completion in the post-test (Figure 5). On the other hand, the haptic training (with no visual cues) results in a decrease in time to task completion in the post-test, showing a positive training effect.

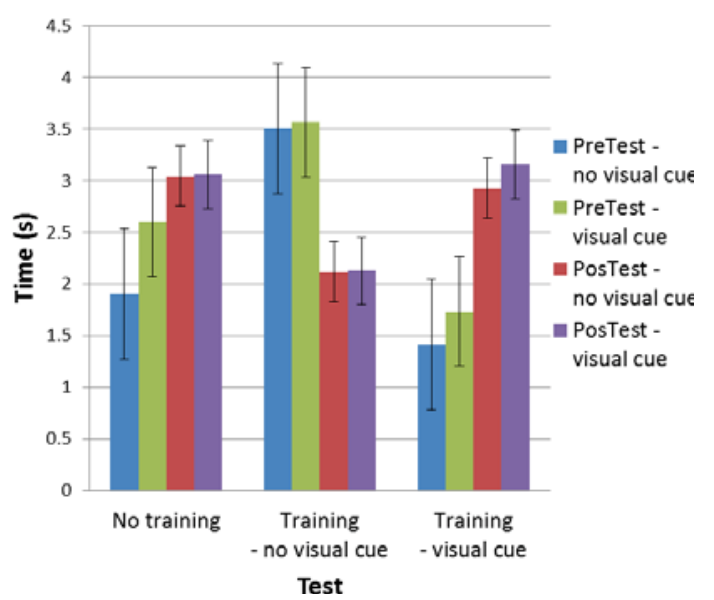

Fig. 5: Average time to task completion

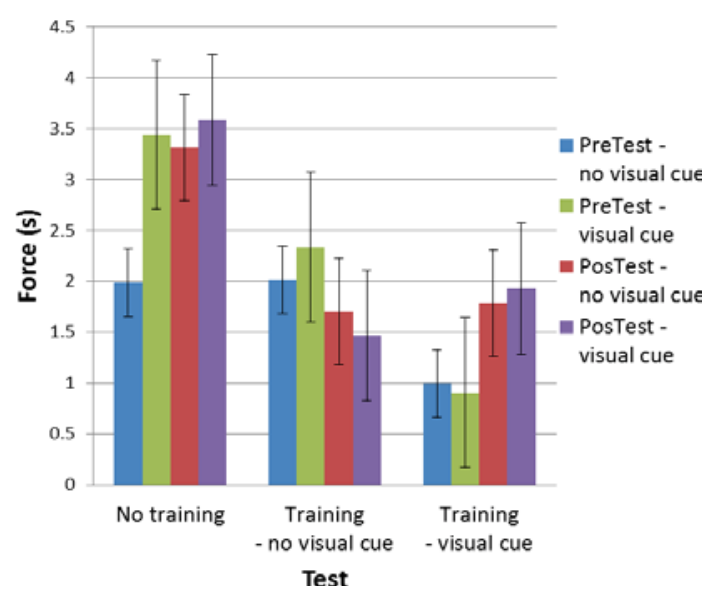

Fig. 6: Average Maximum Force 
A similar pattern can be observed for the maximum force applied by the participants in each group (Figure 6). Indeed, the results show that the visual training did not reduce the amount of applied forces in post-test while the haptic training did.

\section{Discussion and future work}

When the participants received the visual aid for the first time, they could see the direct link between the force they applied and the graph plotted on the screen. However, they did not seem to understand the relationship between the crossing of the layers and the observed or sensed force since no explanation of how to interpret the visual aid was given during the first trial. Nevertheless, some participants were quick to understand the relationship after a few samples and were able to improve their performance. In the case of the novices without training, some understood the visual aid in the wrong way which made them have a worse performance during the test after receiving the visual aid for the first time.

In general, the time required to complete the task decreased as experience was gained with each subsequent trial in the conditions without visual aid. However, when using a visual aid, the time to task completion was always greater than when the participants only followed their haptic perception.

At present, the preliminary analysis does not include the data from expert participants. Therefore, the differences between experts and novices in haptic perception are not known. The effect of training as administered in this study may not reflect that of experience gained through years of practice.

The protocol followed during this study presents a new way of training haptic perception in a needle insertion procedure using a visual cue. It also analyzes the effects of this multimodality training and the results that are obtained when the added modality is removed.

Using a visual cue to train haptic perception does largely improve the precision results when the visual cues are present Error! Reference source not found. However, once this aid is removed, subjects seem to be less certain about the task than before the training session. This can be explained by the fact that the provided visual cues disturbed the participants. In this case, they were relying on their visual perception and not paying enough attention to the haptic feedback.

On the other hand, the participants that did not have visual cue training showed a preference to ignore the visual aid by focusing their attention on the needle and the task. Some participants simply closed their eyes to focus on their haptic perception. By doing so, they performed equally well with or without the visual cue.

Additional experimental sessions are actually conducted with experts. The experts' data will be compared with novices' results in order to determine whether the years of practice can improve significantly the haptic sensitivity for a needle insertion task.

The small participants' sample limits the impacts of our experimental results. The next step will be to run the experiment with a larger participants' sample. A complete statistical analysis of the data is expected to reveal more conclusive results.

\section{Acknowledgements}

This work was supported in part by an award of Chaire Régionale de Chercheur Étranger de la Région Pays de la Loire. The authors are grateful to the grad students of Ecole des Mines de Nantes, and the EMARO students at the Institut de Recherche en Communications et Cybernétique de Nantes, France.

\section{References}

[1] O. Gerovich, P. Marayong, and A. M. Okamura. The effect of visual and haptic feedback on computerassisted needle insertion. Computer Aided Surgery: Official Journal of the International Society for Computer Aided Surgery, 9(6):243-249, Apr. 2004.

[2] M. Zhou, J. Perreault, S. D. Schwaitzberg, and C. G. L. Cao. Effects of experience on force perception threshold in minimally invasive surgery. Surgical Endoscopy, 22(2):510-515, Aug. 2007.

[3] M. Zhou, J. Perreault, S. D. Schwaitzberg, and C. G. L. Cao. Shifts in force perception threshold in laparoscopic surgery with experience. Proceedings of the $51^{\text {st }}$ Annual Meeting of the Human Factors and Ergonomics Society, 636-640, Sept. 2007.

[4] RTV EC00 translucide, Esprit Composite, 14/7/2011. $\mathrm{http} / / / \mathrm{www}$. espritcomposite.com/images/pdf_fiches_tec hniques/EC00ft.pdf

[5] Stern, Melissa K. and Johnson, James H. Just Noticeable Difference. John Wiley \& Sons, Inc. 2010

[6] S.C. Wong, C. Dumas, C. Grosdemouge, C.G.L. Cao. Human sensitivity in compliance evaluation in a toolwielding paradigm. Proceedings of the 55th Annual Meeting of the Human Factors and Ergonomics Society (HFES 2011), September 19-23, 2011. 